\title{
Misclassification of coronary heart disease in mortality statistics. Evidence from the WHO-MONICA Ghent-Charleroi Study in Belgium
}

\author{
S De Henauw, P de Smet, W Aelvoet, M Kornitzer, G De Backer
}

\begin{abstract}
Study objective-To validate the Belgian vital statistics for coronary heart disease (CHD) on the basis of an independent acute myocardial infarction (AMI) register, carried out as part of the WHOMONICA project.

Design-Records of fatal cases of AMI in the WHO-MONICA register were individually linked to the corresponding death certificates.

Setting-Since 1983, the WHO-MONICA Collaborating Centre Ghent/Charleroi registers all fatal and non-fatal AMI in the age group 25-69 years in two geographical areas, Ghent in the northern Dutch speaking part and Charleroi in the southern French speaking part of Belgium. Registration is done according to the MONICA protocol. The official vital statistics in Belgium are published on a yearly basis. They are essentially a reflection of the "underlying" causes of death, coded according to the 9th revision of the International Classification of Diseases (ICD). The study was undertaken in the period 1983-1991.
\end{abstract}

Main results-Out of a total of 741 (Ghent) and 934 (Charleroi) well documented MONICA fatal cases of AMI, 492 $(66.4 \%)$ and $641(68.6 \%)$, respectively, were officially labelled as CHD (ICD code 410-414); 438 (59.1\%) and 385 (41.2\%), respectively, were officially labelled as AMI (ICD code 410). A substantial fraction of the MONICA AMI cases-27.1\% in Ghent and $38.2 \%$ in Charleroi-was coded as "other forms of CHD" (ICD 411-414) or as "other forms of heart disease" (ICD 420-429). The remaining MONICA AMI cases-13.8\% in Ghent and $20.6 \%$ in Charleroi-were classified in either very aspecific (for example, atherosclerosis, ICD 440) or totally unrelated ICD codes (for example, neoplasm, ICD 140-239). Conclusions-It is concluded from the results in this paper that a substantial part of all deaths caused by CHD in Belgium are labelled with incorrect ICD codes and are therefore misclassified in the official mortality statistics for Belgium. This is partly caused by a "drainage" of cases towards less specific CHD related ICD categories. A considerable fraction, however, seems to be absolutely misclassified. (F Epidemiol Community Health 1998;52:513-519)
The availability of reliable cause specific mortality statistics is of crucial importance in the public health domain. Mortality statistics and cause specificity patterns are being usedboth on national and international level — as an indicator for disease end points in public health research and as decision orienting starting points in the development of public health programmes.

However, the quality of these vital statistics has in the past been questionned by several authors for many western countries. ${ }^{1-14}$ The type of investigation this doubt or scepticism is based on, varies and with it also the nature and the certainty of the conclusions. There is quite some variation in the study design. Direct comparisons of death records with hospital records or necropsy records, or both, comparisons of official death records with duplicate death records generated by a panel of independent investigators, and the use of so called "case histories" seem to be the most commonly used methods.

Although growing interest in public health in general will probably stimulate new initiatives to improve the vital statistics, it remains necessary to do a quality control on a regular basis. This issue of quality control is probably more problematic in a number of southern-and western-European countries, where the privacy of a person is very rigidly regulated by law.

A good opportunity for validating cause specific vital statistics is offered by disease registers, as these can be considered independent procedures for tracing specific diseases and should therefore parallel the proces of official death certification and the subsequent coding.

Recently, Tunstall-Pedoe and colleagues ${ }^{15}$ have demonstrated that the agreement between the official routine mortality statistics for coronary heart disease (CHD) and the independent WHO-MONICA acute coronary event register is in general better in populations with high CHD mortality. Their work also showed that in a number of countries (including Belgium) the official statistics for CHD substantially underestimate the CHD mortality as observed in the MONICA register.

The overall quality of the Belgian vital statistics has in the past been shown to be rather low. ${ }^{10-18}$ In this paper, the validity of the vital statistics for CHD is studied for Belgium in more detail on the basis of a record linkage with a population based register for acute coronary events, a part of the WHO coordinated MONICA project. 


\section{Methods}

Data from two distinct databases are compared: data from the Belgian vital statistics and data from the MONICA acute coronary event register. Quite evidently, the comparisons refer to the same time period-1983 until 1991and to the same demographic entities - that is, the populations from the Belgian cities of Ghent and Charleroi.

VITAL STATISTICS

Vital statistics are essentially a reflection of the medical information noted on death certificates by medical doctors (MD). In Belgium, every registered $\mathrm{MD}$ is entitled to complete a death certificate; this death certificate invites to provide information on both the "immediate" and the "underlying" cause of death.

For the period reported in this paper (19831991), the coding of death certificates was done separately in each of the nine Belgian provinces according to the Manual of the International Classification of Diseases-9th revision. ${ }^{19}$

Cause specific mortality statistics are published on a yearly basis by the National Institute of Statistics. These statistics are essentially a reflection of the "underlying" causes of death only, as these are considered "to initialise the train of events that are ultimately leading to a person's death". ${ }^{19}$

In this paper, the terms "a-code" or "b-code" are used to refer to the official ICD code for respectively the "immediate" and the "underlying" cause of death.

MONICA

MONICA is an acronym for "Multinational Monitoring of Trends and Determinants in Cardiovascular Diseases". Its design and methodology have extensively been described elsewhere. ${ }^{2021}$ Essentially, this multinational project studies trends in CHD mortality and morbidity and in coronary risk factors according to a standardised protocol. Validation of vital statistics is one of the marginal objectives of this project.

In this paper, data from the Belgian MONICA centre Ghent-Charleroi are presented. From 1983 until 1991 two geographical areas were monitored for both fatal and non-fatal acute coronary events according to a standardised central protocol; Ghent in the northern Dutch speaking part of Belgium (in the province East Flanders) and Charleroi in the southern French speaking part (in the province Hainault). Events are considered fatal if they result in a person's death within 28 days from the first onset of symptoms of disease.

In these two areas, all "acute medical events" in the population aged 25-69 years were thoroughly investigated. On the basis of the available medical information, fatal events were classified in one of the MONICA categories (see later). For out of hospital fatal events, contacts were made with the MD who completed the death certificate or with the patient's family doctor, or with the emergency team that eventually attended the event, or all three. For hospitalised events with fatal outcome, detailed medical information was obtained from hospital records and from the hospital doctors. If necessary, the family doctor was also contacted.

The criteria and procedures that are used in MONICA to categorise acute events in different categories have been published..$^{152}$ Briefly, data on the clinical picture in the acute phase of the event, on electrocardiographic changes, on serum enzymes, on medical antecedents and on necropsy are, if available, used to classify every acute medical event in one of the following four mutually exclusive categories: (a) no acute myocardial infarction (AMI); (b) definite AMI; (c) possible AMI; (d) fatal events with insufficient data (sometimes also called "unclassifiable").

Fatal acute coronary events are called "definite" if they present with at least either a diagnostic progression of Minnesota codes on serial ECGs or with serum cardiac enzyme concentrations at least twice the limit of normal or with, at necropsy, coronary artery findings of acute coronary occlusion, or all three.

"Possible" fatal coronary events must present with suggestive symptoms or with a documented history of CHD or with suggestive necropsy findings, in the absence of any indication for a cause of death other than CHD, or all three.

"Fatal events with insufficient data" are fatal cases, for which no necropsy data and no data on symptoms or on personal history of CHD are available and for which no other (than CHD) cause of death could be demonstrated.

In this paper, the essential question that is considered is: "In the areas under study, what proportion of the acute coronary events registered in MONICA as 'definite' or 'possible' is missing in the corresponding vital statistics?"

For this purpose, comparisons were made on individual level, linking all MONICA (definite and possible) AMI cases with the ICD codes on the official death records. However, for persons who died outside the areas under study, access to the official ICD codes was impossible; hence, these records were unavailable.

The MONICA "fatal cases with insufficient data" have been excluded from the analyses (except for table 5) as it is appreciated that these cases are characterised by a substantially lower overall diagnostic probability. Inclusion of these cases would therefore attenuate the validity of the inferences made on the basis of these analyses.

Most of the tables in this paper are purely descriptive and do not require any analytical statistical approach. The degree of agreement beyond that expected by pure chance between the MONICA register and the official statistics is measured on the basis of the $\kappa$ statistic. ${ }^{23}$

\section{Results}

Table 1 shows the proportional mortality for selected groups of causes of death as derived from the official vital statistics, for the whole of Belgium, for the two linguistic communitiesFlanders and Wallonia-and for the 
Table 1 Proportional mortality (\%) for selected causes of death in Belgium, the regions Flanders and Wallonia and the cities Ghent and Charleroi (male and female population aged 25-69 years, 1988)

\begin{tabular}{|c|c|c|c|c|c|c|c|c|c|c|c|}
\hline \multirow[b]{2}{*}{ Diseases } & \multirow{2}{*}{$\begin{array}{l}\text { ICD } \\
\text { code }\end{array}$} & \multicolumn{2}{|c|}{ Belgium } & \multicolumn{2}{|c|}{ Flanders } & \multicolumn{2}{|c|}{ Wallonia } & \multicolumn{2}{|c|}{ Ghent } & \multicolumn{2}{|c|}{ Charleroi } \\
\hline & & $M$ & $F$ & $M$ & $F$ & $M$ & $F$ & $M$ & $F$ & $M$ & $F$ \\
\hline All CVD & $390-459$ & 29.4 & 25.6 & 30.2 & 26.4 & 28.6 & 26.1 & 29.6 & 24.4 & 25.6 & 26.0 \\
\hline All IHD & $410-414$ & 14.6 & 9.0 & 15.3 & 9.4 & 13.8 & 8.7 & 13.0 & 8.7 & 11.3 & 9.0 \\
\hline $\mathrm{AMI}$ & 410 & 9.9 & 6.1 & 11.9 & 7.5 & 7.5 & 4.8 & 11.4 & 8.3 & 7.4 & 4.3 \\
\hline CerebroVD & $430-438$ & 4.2 & 6.2 & 4.4 & 6.8 & 4.0 & 5.5 & 4.2 & 4.0 & 3.6 & 5.2 \\
\hline Neoplasms & $140-239$ & 33.8 & 41.3 & 37.4 & 45.1 & 29.7 & 35.5 & 38.4 & 47.3 & 26.8 & 31.3 \\
\hline
\end{tabular}

CVD: cardiovascular disease; IHD: ischaemic heart disease; AMI: acute myocardial infarction; CerebroVD: cerebrovascular disease.

Table 2 Distribution of fatal cases registered in MONICA Ghent-Charleroi across the MONICA diagnostic categories (1983-1991)

\begin{tabular}{|c|c|c|c|c|}
\hline & \multicolumn{2}{|l|}{ Ghent } & \multicolumn{2}{|c|}{ Charleroi } \\
\hline & Number & $\%$ & Number & $\%$ \\
\hline "Definite" AMI & 190 & 13.3 & 231 & 12.4 \\
\hline "Possible" AMI & 611 & 42.6 & 813 & 43.5 \\
\hline \multicolumn{5}{|l|}{ Fatal event with } \\
\hline insufficient data & 632 & 44.1 & 823 & 44.1 \\
\hline Total & 1433 & 100 & 1867 & 100 \\
\hline
\end{tabular}

AMI: acute myocardial infarction.

geographical areas Ghent and Charleroi. The figures in table 1 are data for the 1988 population aged 25-69 years. $^{24}$

According to these official statistics, the proportional mortality for ischaemic heart disease (IHD) in Belgium is-like in most industrialised countries - high in both men and women in this age group.

The overall picture from this table is that for most groups of causes of death, the only differences in proportional mortality are found between sexes, not between regions. In general, the picture for Ghent and Charleroi is very comparable to that of the community where these cities are situated in geographically, respectively Flanders and Wallonia.

Regarding the completeness of the official mortality statistics, there are no reasons for any doubt not only for the whole of Belgium but also for the smaller administrative subunits of the country.

In table 2 the distribution of the MONICA registered cases across the MONICA diagnostic categories is presented. This shows a very similar picture for the two centres. The category "definite" is clearly the smallest fraction as, evidently, a large proportion of fatal cases is either medically unattended or poorly documented and therefore falls predominantly in the categories "possible" or "fatal events with insufficient data".

As explained in the methods, the ICD codes for a small fraction of the MONICA fatal cases who died outside the areas under study were not available. Sixty such cases (for Ghent) and 110 cases (for Charleroi) were classified as "definite" or "possible" MONICA AMI cases. These cases as well as the MONICA cases categorised as "fatal events with insufficient data" were excluded from the analyses. Hence, the study population for the tables 3 and 4 and for figure 1 comprises 741 MONICA cases for Ghent and 934 cases for Charleroi.

Table 3 gives the proportion of the MONICA "definite" and "possible" AMI cases that were officially coded as coronary heart disease (the term used in the ICD manual is ischaemic heart disease, codes 410-414). Evidently, the match between MONICA and death certificate codes is highest at the top of this table, taking into account the broader ICD category $410-414$ on either the a-code or the b-code. As the comparison is refocused in a stepwise way towards more accurate codes and taking into account only the "underlying" cause of death, there is a gradual decrease in the proportional match between the two systems.

Inclusion of the MONICA category "fatal events with insufficient data" results in a considerable further decrease in the match between the two systems (data not shown). These figures are, however, not used for further interpretation, as it is appreciated that they refer to a less homogeneous MONICA dataset with substantially lower overall diagnostic probability.

Of all MONICA definite and possible AMI cases, 33.6\% (Ghent) and 31.4\% (Charleroi) are not coded "CHD" for the "underlying" cause on the death certificate and are consequently not included in the official statistics for CHD. Two decisive steps further down the precision tree (table 3), taking into consideration that what is registered in MONICA should be coded as ICD code 410 for the "underlying" cause, it seems that $40.9 \%$ (Ghent) and $58.8 \%$ (Charleroi) of the MONICA cases are not included in the official - on a national and international level published-mortality statistics for AMI.

Where are these "misclassified" cases categorised in the official vital statistics? The answers for this question are presented in figure 1 and table 4 .

Figure 1 shows the proportional distribution of the official ICD b-codes for all "definite" and "possible" MONICA cases for Ghent and Charleroi. For those MONICA cases officially not b-coded as AMI, the proportional distribution of their official ICD b-codes is then shown in some more detail in table 4.

A large part of the "misclassified" fraction is in the official statistics picked up by other more or less related categories, although still a considerable fraction is classified in either very aspecific or totally CHD unrelated categories. Altogether $66.2 \%$ (Ghent) and $65.0 \%$ (Charleroi) of the MONICA cases not officially

Table 3 Proportion of MONICA "definite" and "possible" AMI cases ( $n=741$ for Ghent; $n=934$ for Charleroi) officially coded as IHD (ICD 410-414)

\begin{tabular}{llllll}
\hline & \multicolumn{2}{l}{ Ghent } & & \multicolumn{2}{l}{ Charleroi } \\
\cline { 2 - 3 } \cline { 5 - 6 } MONICA cases with & Number & $\%$ & & Number & $\%$ \\
\hline 410-414 on "a" or & & & & \\
"b"-code & 503 & 67.9 & & 724 & 77.5 \\
410-414 on "b"-code & 492 & 66.4 & & 641 & 68.6 \\
410-411 on "b"-code & 444 & 59.9 & & 463 & 49.6 \\
410 on "b"-code & 438 & 59.1 & & 385 & 41.2
\end{tabular}

"a"-code: "immediate" cause of death; "b"-code: "underlying" cause of death; 410-414: ischaemic heart disease; 410: acute myocardial infarction; 411: Other acute and subacute forms of ischaemic heart disease; 412: old myocardial infarction; 413: angina pectoris; 414 : other forms of chronic ischaemic heart disease. 

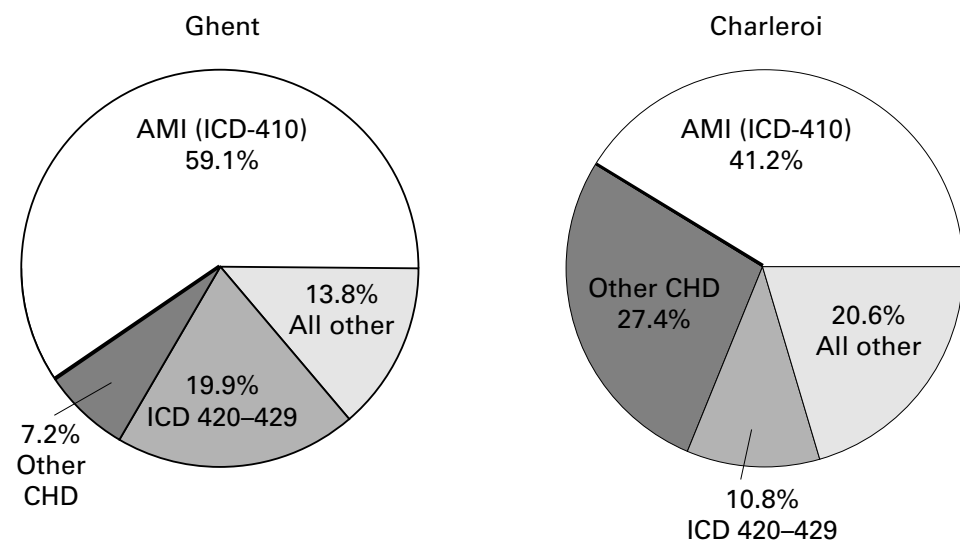

Figure 1 Proportional distribution of official ICD b-codes (underlying cause of death) for the MONICA "definite" and "possible" AMI cases. ICD 420-429: other forms of heart disease. "All other": all other iCD codes. "Other CHD": ICD 411-414.

Table 4 MONICA "definite" or "possible" AMI cases, officially not coded as " 410 "*: proportional distribution of their official ICD codes*

\begin{tabular}{llll}
\hline ICD code & ICD definition & $\begin{array}{l}\text { Ghent } \\
(n=303)\end{array}$ & $\begin{array}{l}\text { Charleroi } \\
(n=549)\end{array}$ \\
\hline $411-414$ & IHD exclusive of AMI & 17.6 & 46.6 \\
$420-429$ & Other forms of heart disease & 48.6 & 18.4 \\
427.4 & Ventricular fibrillation/flutter & 3.3 & 2.7 \\
427.5 & Cardiac arrest & 12.5 & 0.5 \\
427 & Cardiac arrhythmias & 19.3 & 4.9 \\
428 & Heart failure & 5.9 & 6.5 \\
785 & Symptoms involving cardiovascular system & 1.6 & 0.9 \\
$798-799$ & Sudden death, cause unknown. Other ill & 4.6 & 5.1 \\
& defined and unknown causes & & \\
Rest† & & 27.6 & 28.9 \\
Among these: & & & \\
$140-208$ & Malignant neoplasm & 1.6 & 2.2 \\
250 & Diabetes mellitus & 2.9 & 2.7 \\
$430-438$ & Cerebrovascular disease & 2.9 & 2.0 \\
440 & Atherosclerosis & 1.6 & 3.3 \\
491 & Chronic bronchitis & 5.9 & 0.3 \\
$401-405$ & Hypertensive disease & 3.3 & 1.6 \\
& All other codes & 9.4 & 16.8 \\
\hline
\end{tabular}

*Underlying cause of death. †Including more than 20 codes. Data shown as percentages.

b-coded as 410 are covered by the broad category " $\mathrm{CHD}$ other than $\mathrm{AMI}$ or other forms of heart disease". A remarkable region differential result must be noted here as in Charleroi $46.6 \%$ is picked up by the category " $\mathrm{CHD}$ exclusive of AMI", while in Ghent this is only $17.6 \%$. In Ghent, on the other hand, $48.6 \%$ are found as "other forms of heart disease" while this category accounts for a much smaller fraction in Charleroi $(18.4 \%)$. It looks as if a mirror image-like misclassification is taking place in the two respective regions, a phenomenon for which no plausible explanation is available.

Another $6.2 \%$ (Ghent) and 6.0\% (Charleroi) of the officially missing MONICA AMI cases are picked up by the combination of the categories "symptoms involving the cardiovas-

Table 5 Classification of deaths according to the official CHD statistics and according to the MONICA register (1983-1991); degree of agreement between the two systems, calculated on the basis of the $\kappa$ statistic

\begin{tabular}{|c|c|c|c|c|c|c|}
\hline & \multicolumn{6}{|c|}{ Official death register } \\
\hline & \multicolumn{2}{|c|}{ ICD 410-414 } & \multicolumn{2}{|c|}{ All other ICD codes } & \multicolumn{2}{|l|}{ Total } \\
\hline & Ghent & Charleroi & Ghent & Charleroi & Ghent & Charleroi \\
\hline \multicolumn{7}{|l|}{ MONICA } \\
\hline Fatal AMI & 751 & 999 & 682 & 868 & 1433 & 1867 \\
\hline $\begin{array}{l}\text { Death caused by other } \\
\text { causes }\end{array}$ & 84 & 100 & 5798 & 6277 & 5882 & 6377 \\
\hline Total & 835 & 1099 & 6480 & 7145 & 7315 & 8244 \\
\hline
\end{tabular}

к Statistic: for Ghent: 0.61 (95\% CI 0.58, 0.63). Charleroi: 0.61 (95\% CI 0.59, 0.63). cular system" and "sudden death, cause unknown and other ill-defined and unknown causes of mortality".

Finally, the rest of table 4 allows to conclude that a large proportion of those deaths, which are picked up by the MONICA register with a high diagnostic probability but not officially labelled " 410 ", have been given a very aspecific official code (for example, atherosclerosis, ICD code 440) or even a code that is totally unrelated to CHD (for example, neoplasms, ICD codes 140-208)-altogether $27.6 \%$ in Ghent and $28.9 \%$ in Charleroi.

The MONICA register and the official death register are actually both "measuring" the same phenomenon-that is, death caused by a disease, in this case, CHD. Both measurement systems are characterised by their own sensitivity and specificity, which can however not be calculated in the absence of a golden standard. The $\kappa$ statistic measures the degree of agreement between two measuring systems and expresses to what extent this agreement accounts for the difference between a theoretical maximal agreement (in which the two are measuring in exactly the same way) and a minimal agreement on a simple chance basis. ${ }^{23}$

Table 5 shows the data that are used for calculating the $\kappa$ statistic with the corresponding results. The MONICA category "fatal events with insufficient data" is included for these calculations as it also contains cases with ICD code 410-414 on their death certificate. A moderately low $\kappa$ statistic of 0.61 was found in both Ghent and in Charleroi. Apparently, the congruence between the two measuring systems is only capable of filling $61 \%$ of the theoretical gap between a perfect agreement and an agreement by chance. This rather low agreement is in line with the findings reported in tables 3 and 4 and figure 1 .

\section{Discussion}

The validity of cause specific mortality statistics varies in time, across countries, across disease groups, across age groups, etc, and this can evidently jeopardise the interpretation of the observations relying on them..$^{1-14}$

The required accuracy of the mortality statistics may, to some extent, depend on the purpose for which they are being used. Joossens et $a l^{25}$ have, for example, stated that, for CHD trend analysis, even a considerable degree of inaccuracy of death certification causes no interpretational problems, provided that the misclassification occurs on a random basis. In contrast, Stehbens ${ }^{26}$ concludes-on the basis of an exhaustive review of literature from 1919 to 1985 - that the validity of the observed temporal changes in official CHD mortality must thoroughly be questionned and can in no way be used for any scientific purpose. Kelson et $a l,{ }^{27}$ Grulich et $a l^{28}$ and Hoel et $a l^{29}$ come to similar conclusions regarding trends in cancer mortality. Whatever the potential effect of inaccuracies in vital statistics on time trends, it certainly is very straightforward when it comes for instance to depicting the prevalence of diseases among population groups or when it comes to ecological analysis, 
where misclassification - whatever the underlying mechanism - can always bias the results and the concomitant conclusions.

The need for improving the quality of vital statistics has in fact long been recognised, already during the first half of this century ${ }^{30} 31$ and increasingly so after the second world war as the use of these data for international comparisons and trend analyses became more and more widespread. ${ }^{32}{ }^{33}$ The main reason for the WHO MONICA study was also the need for more precise data to better understand the observed changing trends in official mortality data. One of the marginal objectives, formulated from the very beginning of this study, was the possibility of validating the vital statistics. Tunstall-Pedoe and colleagues ${ }^{15}$ have recently demonstrated that for a number of participating MONICA centres, the vital statistics represent a considerable underestimation of the occurrence of AMI as registered in MONICA. This phenomenon was particularly prominent for the participating centres from France, Denmark, Poland, and also Belgium.

The differences between the official vital statistics for CHD and the MONICA register as described in this paper are large (see table 3) and the degree of agreement is low (table 5). Clearly, either one of these two systems or both are burdened with critical problems of accuracy of registration.

The MONICA register is not considered as the gold standard with respect to the comparisons made in this paper. It is assumed, though, that the MONICA register has a high sensitivity, given the very broad first entrance for potential cases. The specificity, on the other hand, might show some unavoidable deficiencies; this relates predominantly to the natural history of the fatal form of this disease, for which in many cases not much hard evidence can be obtained. However, by excluding the category "fatal events with insufficient data" from part of the analyses, a high overall diagnostic probability is achieved and should guarantee a low number, if any, of false positive cases in the dataset.

Clear evidence for the idea that the MONICA register gives a closer approximation of reality and that it is indeed the official register that is defective with respect to AMI registration, is given by a number of considerations concerning the methodology of these two systems.

The MONICA protocol for ascertainment of the different categories for acute coronary events provides algorithms with inclusion and exclusion criteria that have been validated extensively. ${ }^{34}$ Moreover, every participating centre is also subject to external quality control on the basis of blinded test cases, which gave satisfactory results for our centres.

In MONICA, every acute medical event has to be classified in one of four unambiguous, mutually exclusive, categories. The official register, on the other hand, starts from an open entry on the death certificate in which the certifying doctor, although asked to give specific medical information, is not actually forced to make a conclusion on the cause of death in terms of a sharply defined clinical disease entity.

Quite a large proportion of the official b-codes of the MONICA definite and possible AMI cases are referring to other forms of IHD or to symptoms or complications of AMI, rather than to the disease itself $(27.1 \%$ in Ghent and $38.2 \%$ in Charleroi, see figure 1). This phenomenon can be considered as "relative misclassification", with underlying causes of death and immediate causes of death as well as disease symptoms being mixed up within a large frame of a disease group, for example, cardiovascular diseases. The most typical examples in this study were the coding of AMI as "ventricular fibrillation" (ICD code 427.4) or "heart failure" (428) on the b-code (see table 4). If a number of AMI cases are indeed classified under the label of one of their potential complications and to the extent that the latter can also be complications of other disease(s), this leads to an irreversible loss of information with respect to the occurrence of individual diseases involved. Of much larger concern still are the results regarding "absolute misclassification". In Ghent $13.8 \%$ and in Charleroi $20.6 \%$ (see fig 1 ) of the MONICA definite and possible AMI cases were "lost" in categories that are either very aspecific (for example, atherosclerosis) or have not got anything to do with AMI or CHD (for example, neoplasms).

Given the fact that the access to the death certificate was limited to the ICD codes (generated by the coding teams on the basis of the medical information written by the certifying MD), it was impossible to elucidate to what extent misclassifications were caused by problems in certification compared with coding, or a combination of both.

In the areas under study, a large proportion of deaths attributed to CHD (ICD 410-414) with high diagnostic probability by the MONICA procedures is not included in the official CHD statistics $(33.6 \%$ in Ghent and $31.4 \%$ in Charleroi, see fig 1 and table 3 ). There are no reasons to assume that this misclassification problem would not be present in the rest of Belgium as well. To what extent this misclassification of AMI cases (according to MONICA) also leads to an underestimation of the true CHD mortality by the official mortality statistics, falls beyond the scope of this paper. The comparison of the total numbers of CHD deaths registered in the official mortality statistics ( $n=835$ for Ghent and $n=1099$ for Charleroi, data not shown) with the total number of $\mathrm{CHD}$ cases registered in the MONICA study (including "fatal events with insufficient data", $n=1433$ for Ghent and $\mathrm{n}=1867$ for Charleroi, see table 2) would suggest an underestimation of CHD mortality by the official statistics. This can however not be a definite conclusion from this paper as, by including the "fatal cases with insufficient data" in the database, the specificity of the MONICA register probably falls below critical quality standards and therefore the above comparison would not be valid. 
It should be noted that the two systems for registration of disease under consideration in this paper are not entirely independent of each other, as the MONICA register makes use of the death certificates to trace potential cases. However, the decision whether or not a particular case is included in the MONICA register is to the largest possible extent based on an independent procedure of case ascertainment, using information provided by the family doctor, the hospital doctor, the emergency team, etc. Only in a very limited number of cases is the decision of including cases in the MONICA register based solely on information from the death certificate.

Our results and conclusions apply, evidently, only to the age group 25-69 years. The accuracy of the vital statistics in older age groups could be even lower as the clinical picture preceding death is often much more complicated in older people. ${ }^{35}$

Only a limited number of studies looking at the validity of CHD vital statistics, is actually population-based.

In the Dutch speaking part of Belgium, inter-provincial general coding differences have been reported by Aelvoet et al. ${ }^{17}$

In a study of 21 million death records in the USA in the period 1968-1978, Gittelsohn ${ }^{36}$ found an increasing general tendency towards less specific codes, characterised by a strong between state variation. The proportion of acute ischaemic events within the total group of IHD ranged from one third in one state to nearly $80 \%$ in another, strongly suggestive for a differential labelling practice among physicians from one state to another, although differing coding practices can also be involved. This picture compares strongly to the remarkable and unexplained between region difference that was found in this study in the ratio of the groups "other forms of heart disease" to "CHD other than AMI" for misclassified cases.

In an Australian study comparing official death records with data obtained through an independent heart attack surveillance study in 1979, Dobson et $a l^{37}$ conclude that fatal cases of AMI are fairly well covered by the broad category of IHD in the Australian official statistics. Only $10 \%$ of the registered AMI cases were not officially coded within the ICD range 410-414 (contrasting sharply with $33.6 \%$ and $31.4 \%$ in respectively Ghent and Charleroi in this study).

Results comparable to ours were reported in a more recent paper by Ron $e t a l^{13}$ looking at the agreement between death certificate and necropsy diagnoses in a population of atomic bomb survivors, in which the detection and the confirmation rate for heart and other vascular diseases was only between $50 \%$ and $70 \%$

In general, the most frequently used methods for validating vital statistics are sensitive to bias. Selection bias can occur in studies linking death certificates to premortem or postmortem medical records as these studies are mostly based on in hospital deaths and not on a general population. Reporting bias can be expected in studies using so called "case histories" that are sent to physicians or coders, or both, and are thus brought under special attention of these panels, in contrast with a routine act of certification or coding.

This study has the advantage of being population-based-and thus free of selection bias - and of reporting on a real situation using existing death certificates, hence free from reporting bias.

The model of death certificate currently used in Belgium does not include a section on "contributory" cause of death and provides only very limited space for the "immediate" and "underlying" cause of death. Adoption of the hierarchical model proposed by the $\mathrm{WHO}^{19}$ could yield more comprehensive information on the premortem and postmortem medical findings and could therefore lead to more accurate coding. ${ }^{38-40}$

It has been shown that many physicians do not sufficiently know what exactly they are expected to put on the document and how to do it. ${ }^{41-46}$ Therefore, the topic of death certification should be highlighted more emphatically during the medical training, both in theoretical courses and during the hospital residency. ${ }^{47}{ }^{48}$ Instruction guides accompanying the death certificate can also be useful. ${ }^{44} 46$

For epidemiological purposes, so called "multiple cause of death" data, ${ }^{49-51}$ in which the immediate and contributory conditions as well as the underlying causes are taken into account simultaneously, should often preferably be used. Multiple cause of death statistics provide a more complex, more realistic and more reliable picture; they are particulary useful for studying diseases that can in official records easily be masked by their complications or symptoms as it is the case for AMI.

\section{Addendum}

Since 1993, the coding of the death certificates has been done centrally in the Dutch speaking part of Belgium. There are already indications that this will favourably influence the quality of the vital statistics (W Aelvoet, Ministry of Health for the Flemish Community, personal communication), but this has not been studied systematically yet. Quality control of death certification and coding on a regular basis remains however indispensable.

Funding: this research was supported by grant no 3.9002 .84 of the National Research fund.

1 Hunt LW, Silverstein MD, Reed CE, et al. Accuracy of the death certificate in a population-based study of asthmatic patients. FAMA 1993;269:1947-52.

2 Goldacre MJ. Cause-specific mortality: understanding uncertain tips of the disease iceberg. F Epidemiol Community Health 1993;47:491-6.

3 Gittelsohn A, Senning J. Studies on the reliability of vital and health records : I. Comparison of cause of death and hospital record diagnoses. Am f Public Health 1979;69: $680-9$.

4 Kircher T, Nelson J, Burdo H. The autopsy as a measure of accuracy of the death certificate. N Engl F Med 1985;313: 1263-9.

5 Benavides FG, Bolumar F, Peris R. Quality of death certificates in Valencia, Spain. Am f Public Health 1989;79:13524.

6 Andresen EM, Lee JA, Pecoraro RE, et al. Underreporting of diabetes on death certificates, King County, Washington. Am $\mathcal{F}$ Public Health 1993;83:1021-4. 
7 Balkau B, Jougla E, Papoz L. European study of the certification and coding of causes of death of six clinical case histories of diabetic patients. Eurodiab

8 Percy C, Stanek E, Gloeckler L. Accuracy of cancer death certificates and its effect on cancer mortality statistics. $A m$ f Public Health 1981;71:242-50.

9 Neukirch F, Maguin P, Perdrizet S, et al. The accuracy of death certification of respiratory disease in France and seven other countries of the EEC. Rev Mal Respir 1984;1:361-7.

10 Farebrother MJB, Kelson MC, Heller RF on behalf of the EEC Working Party. Death certification of farmer's lung and chronic airway diseases in different countries of the EEC. Br $\mathcal{F}$ Dis Chest 1985;79:352-61.

11 Mackenbach JP, Snels IAK, Friden-Kill LM. Diabetes mellitus als doodsoorzaak. Ned Tijdschr Geneeskd 1991;135: 1492-6.

12 Percy C, Dolman A. Comparison of the coding of death certificates related to cancer in seven countries. Public Health tificates related to can
Rep 1978;93:335-50.

13 Ron E, Carter R, Jablon S, et al. Agreement between death certificate and autopsy diagnoses among atomic bomb surcertificate and autopsy diagnoses a
vivors. Epidemiology 1994;5:48-56.

14 Andersson DK, Svardsudd K. The value of death certification statistics in measuring mortality in persons with diabetes. Scand F Prim Health Care 1994;12:114-20.

15 Tunstall-Pedoe H, Kuulasmaa K, Amouyel P, et al. Myocardial infarction and coronary deaths in the World Health Organization MONICA project. Circulation 1994;90:583612 .

16 Kelson MC, Heller RF on behalf of the EEC Working Party. The effect of death certification and coding practices on observed differences in respiratory disease mortality in 8 EEC countries. Rev Epidemiol Sante Publique 1983;31:423EEC

17 Aelvoet W, Humblet PC, Lagasse R. Het in routine gebruik maken van een geïnformatiseerd controleprogramma als maken van een geinformatiseerd controleprogramma als middel tot kwaliteitsbewaking van de statistieken van de
burgerlijke stand in Vlaanderen. Arch Public Health burgerlijke stan $1991 ; 49: 1-8$.

18 Van Oyen H, Aelvoet W, Tafforeau J. Overlijdenspatroon in het Vlaamse Gewest. Tijdschr voor geneeskunde 1993;49: $821-8$

19 World Health Organisation. International classification of diseases: manual of the international statistical classification of diseases, injuries and causes of death; based on the recommendations of the Ninth Revision Conference, 1975. Geneva:World Health Organisation, 1977 .

20 Böthig S. WHO MONICA Project: objectives and design. Int F Epidemiol 1989;18:S29-37.

21 WHO MONICA Project Principal Investigators (prepared by Tunstall-Pedoe $\mathrm{H}$ ). The World Health Organisation MONICA Project (Monitoring Trends and Determinants in Cardiovascular Diseases): a major international collaboin Cardiovascular Diseases): a major inte
ration. $\mathcal{7}$ Clin Epidemiol 1988;41:105-14.

22 WHO MONICA Project. Monica Manual, revised edition. Geneva:Cardiovascular Diseases Unit,WHO, 1990.

23 Fleiss JL. Statistical methods for rates and proportions. New York: John Wiley, 1981:217.

24 Van Oyen H, Tafforeau J, Drieskens S, et al. Overlijden en oorzaak-specifiek overlijden in de Vlaamse gemeenschap, 1988. Brussel:Centrum voor operationeel onderzoek in volksg ezondheid,IHE, 1994

25 Joossens JV, Kesteloot $\mathrm{H}$. The value of ischaemic heart disease vital statistics since 1968. Acta Cardiol 1989;44:389405

26 Stehbens WE. An appraisal of the epidemic rise of coronary heart disease and its decline. Lancet 1987;i:606-11.
27 Kelson $M$, Farebrother $M$. The effect of inaccuracies in death certification and coding practices in the European mortality statistics. Int $\mathcal{F}$ Epidemiol 1987;16:411-14.

28 Grulich AE, Swerdlow AJ, dos Santos Silva I, et al. Is the apparent rise in cancer mortality in the elderly real? Analysis of changes in certification and coding of cause of death in England and Wales, 1970-1990. Int F Cancer 1995;63: $164-8$.

29 Hoel DG, Ron E, Carter R, et al. Influence of death certificate errors on cancer mortality trends. 7 Natl Cancer Inst 1993;85:1063-8.

30 Cabot RC. Diagnostic pitfalls identified during a study of three thousand autopsies. $\mathcal{F A M A}$ 1912;59:2295-8.

31 Bolduan CF, Bolduan NW. Is the "appalling increase" in heart disease real? F Prevent Med 1932;6:321-33.

32 Gall EA. The necropsy as a tool in medical progress. Bull NY Acad Med 1968;44:808-29.

33 Anonymous. The accuracy and comparability of death statistics. WHO Chronicle 1967;21:11-17.

34 Tunstall-Pedoe H. Diagnosis, measurement and surveillance of coronary events. Int f Epidemiol 1989;18:S169-73.

$35 \mathrm{Kohn}$ RR. Cause of death in very old people. $\mathcal{F A M A} 1982$; 247:2793-7.

36 Gittelsohn AM. On the distribution of underlying causes of death. Am f Public Health 1982;72:133-40.

37 Dobson AJ, Gibberd RW, Leeder SR. Death certification and coding for ischemic heart disease in Australia. Am $\mathcal{F}$ Epidemiol 1983;117:397-405.

38 Crews DE, Stamler J, Dyer A. Conditions other than underlying cause of death listed on death certificates provide additional useful information for epidemiologic research. Epidemiology 1991;2:271-5.

39 Dublin L, Van Buren G. Contributory causes of death. Their importance and suggestions for their classification. Am $\mathcal{F}$ Public Health 1923;13:100-5.

40 Mackenbach JP. Secundaire doodsoorzaken. T Soc Gezondheidsz 1987;65:524-8.

41 Alderson MR, Bayliss RIS, Clarke CA, et al. Death certification. BMF 1983;287:444-5.

42 Leadbeatter S. Semantics of death certification. $\mathcal{F} R$ Coll Physicians Lond 1986;20:129-32.

43 Balarajan R. Certification of death and the clinician. Health Trends 1983;15:53-6.

44 Maudsley G, Williams EM. Death certification by house officers and general practitioners - practice and performance. F Public Health Med 1993;15:192-201.

45 Jordan JM, Bass MJ. Errors in death certificate completion in a teaching hospital. Clin Invest Med 1993;16:249-55.

46 Hanzlick R. Death certificates. The need for further guidance. Am $\mathcal{F}$ Forensic Med Pathol 1993;14:249-52.

47 Kircher T, Anderson RE. Cause of death. Proper completion of the death certificate. $\mathscr{F} A M A$ 1987;258:349-52.

48 Kesteloot H, Joossens JV. Mortaliteit in België. Betekenis van het overlijdenscertificaat. Tijdschr Geneeskunde 1991; 47:795-8.

49 Israel RA, Rosenberg HM, Curtin LR. Analytical potential for multiple cause-of-death data. Am $\mathcal{f}$ Epidemiol 1986;124: 161-79.

50 Chamblee RF, Evans MC. New dimensions in cause of death statistics. Am F Public Health 1982;72:1265-70.

51 Steenland K, Nowlin S, Ryan B, et al. Use of multiple-cause mortality data in epidemiologic analyses: US rate and proportion files developed by the National Institute for Occupational Safety and Health and the National Cancer Institute. Am f Epidemiol 1992;136:855-62. 